\title{
Valuing Economic Impact Reductions of Nutrient Pollution from Livestock Waste
}

\author{
Apoorva M. Sampat, ${ }^{\dagger}$ Andrea Hicks, ${ }^{\ddagger}$ Gerardo J. Ruiz-Mercado,,${ }^{, \uparrow}$ and \\ Victor M. Zavala ${ }^{\dagger}$ \\ $\dagger$ Department of Chemical and Biological Engineering, University of Wisconsin-Madison, 1415 \\ Engineering Drive, Madison, WI 53706, USA \\ $\ddagger$ Department of Civil and Environmental Engineering, University of Wisconsin-Madison, 1415 \\ Engineering Drive, Madison, WI 53706, USA \\ \Office of Research and Development, U.S. Environmental Protection Agency, 26 West Martin \\ Luther King Drive, Cincinnati, Ohio 45268, USA \\ E-mail: ruiz-mercado.gerardo@epa.gov
}

\begin{abstract}
Nutrient pollution from livestock waste impacts both fresh and marine coastal waters. Harmful algae blooms (HABs) are a common ecosystem-level response to such pollution that is detrimental to both aquatic life and human health and that generates economic losses (e.g., property values and lost tourism). Waste treatment and management technologies are not well established practices due, in part, to the difficulty to attribute economic value to associated social and environmental impacts of nutrient pollution. In this work, we propose a computational framework to quantify the economic impacts of HABs. We demonstrate the advantage of quantifying these impacts through a case study on livestock waste management in the Upper Yahara watershed region (in the state of Wisconsin, USA). Our analysis reveals that every excess kilogram of phosphorus runoff from livestock waste results in total economic losses of 74.5 USD. Furthermore, we use a coordinated market analysis to demonstrate that this economic impact provides
\end{abstract}


a strong enough incentive to activate a nutrient management and valorization market that can help balance phosphorus within the study area. The proposed framework can help state, tribes, and federal regulatory agencies develop regulatory and non-regulatory policies to mitigate the impacts of nutrient pollution.

Keywords: livestock waste; economics; eutrophication; phosphorus;

\section{Introduction}

Agricultural non-point nutrient pollution is the leading cause of water quality impairments to rivers, the second largest cause for wetlands, the third largest for lakes, and is a major contributor to the contamination of groundwater. ${ }^{1}$ When excess nutrients (in the form of chemical fertilizers or manure) are applied to croplands having legacy phosphorus in soils and there is either rain or snowmelt following the application, the nutrients runoff to the waterbodies resulting in ecosystem responses such as excess growth of algae. The rapid growth of algae is known as harmful algae blooms (HABs) and the toxins released during HABs can be detrimental to both aquatic life and human health. HAB events can cause massive fish kills, ${ }^{2}$ closure of beaches ${ }^{3}$ and shellfish beds, ${ }^{4}$ death of marine mammals and sea birds, ${ }^{5}$ coral reefs, ${ }^{6}$ and alter marine habitats. ${ }^{7}$ This in turn hurts tourism, recreational and commercial fishing, and valued habitats, that are vital to local economies. ${ }^{8}$ In July 2019, all 21 beaches in the State of Mississippi were closed due to HABs. ${ }^{3}$ Dodds et al. ${ }^{9}$ estimate an average annual loss of 770 million USD in recreational activities due to eutrophication of U.S. freshwaters. In the State of Florida, HABs have resulted in a monthly loss of 2.8 and 3.7 million USD corresponding to restaurant and lodging revenue, respectively. ${ }^{10}$ Frequent occurrence of HABs also lowers property values of lakefront properties. The loss in property values are the largest impact bearers of eutrophication with an estimated average economic loss of 1.6 billion USD annually. ${ }^{9}$

Estimating the scale of economic loses associated to HABs provides valuable information to determine appropriate counter measures to prevent or mitigate the loses. ${ }^{11}$ Unfortunately, not many studies have been conducted to quantify the economic impacts of HABs. Most of the reported stud- 
ies are driven by impacts of toxins in commercial fisheries. ${ }^{12-14}$ Hoagland et al. ${ }^{15}$ first estimated an annual expenditure of 20 million USD in public health due to seafood poisoning caused by HABs in the United States. HABs can also cause respiratory illness such as asthma, pneumonia, and bronchitis. In the gulf coast of Florida, wind causes toxins released by HABs to form aerosols and causes damage to the respiratory system. ${ }^{16}$ For the Saratosa County in the State of Florida alone, the cost of respiratory illnesses associated with HABs is estimated to be 0.5 to 4 million USD annually. ${ }^{17}$ Phaneuf et al. ${ }^{18}$ developed a tool that estimates the monetary impact on recreational use of freshwater lakes in the southeast for a change in water quality. As an input, the tool requires the current and desired concentrations of phosphorus, nitrogen, and chlorophyll a. It also requires an estimate on the number of trips to the lake. It then outputs the total economic impact of improving the water quality from a recreational use perspective. As it will become evident later, our work can provide an extension to this tool by providing a methodology to estimate the impact on the number of trips to the lake as a function of water clarity, estimating the impact on lakefront property values, and quantifying the clean up expenses. Quantifying the impacts of nutrient pollution can also drive the decision-making for recovery processes. Sena et al. ${ }^{19}$ observe that recovering struvite from a waste water treatment plant in Madison, WI is economically viable if we consider the avoided cost of nutrient pollution.

A number of the economic analyses available in literature rely on survey data for estimating the economic impact of algae blooms. ${ }^{9,16}$ Dodds et al. ${ }^{9}$ use data on total phosphorus and nitrogen concentrations in different ecoregions to estimate economic damages of eutrophication in U.S. freshwaters. Fleming et al. ${ }^{16}$ estimate health impacts of red tides in the Saratosa county in Florida through a statistical model that correlates the HABs outbreak with the cost of emergency visits to the Saratosa Memorial Hospital for respiratory illnesses. Such methodologies are difficult to scale to different geographical areas facing similar HABs related issues. A model based on easily measurable quantities (e.g., water clarity) can help extend the model to different geographical locations and provide a preliminary estimate towards quantifying the economic impacts of HABs. Vesterinen et al. ${ }^{20}$ propose a hurdle model to quantify the change in demand for recreational activities as a function of water clarity. The hurdle model is proposed for Finland, but it can be useful in esti- 
The value of lakefront properties depends strongly on water clarity. Dodds et al. ${ }^{9}$ estimate that the property value decreases by $15.6 \%$ for every meter decrease in water clarity (measured by Secchi depth). The Secchi depth (SD) is a metric used for water clarity that is calculated by inserting a black and white colored disc in the water and by measuring the maximum depth until which the disc is 
visible. Algae blooms have a direct impact on water clarity; specifically, a high total phosphorus (TP) concentration during an algae bloom turns the water turbid, reducing the Secchi depth. The relationship between the Secchi depth and total phosphorus concentration is given by: ${ }^{21}$

$$
\ln (\mathrm{SD})=a+b \ln (\mathrm{TP})
$$

89

where SD is the Secchi depth in meters and TP is the total phosphorus concentration in $\mu g / L$. Parameters $a$ and $b$ depend on the lake type. For stratified natural lakes (e.g. Lake Mendota, WI), $a=2.10$ and $b=-0.44 .^{21}$

\section{Recreational Costs}

A decrease in water clarity also reduces the demand for recreation activities such as swimming and fishing. ${ }^{23}$ Vesterinen et al. ${ }^{20}$ propose a hurdle model to quantify the change in demand for recreational activities as a function of water quality. This hurdle model is proposed for the waterbodies in Finland. Currently, no other relevant studies exist that quantify the impact of water quality on recreational activities. Also, we can apply this model for the State of Wisconsin based on the observation that both Finland and Wisconsin have similar population sizes and similar median household income, and both face problems of eutrophication of water bodies. ${ }^{23}$ In fact, this model is used by the Wisconsin Department of Natural Resources.

The hurdle model is a two stage model: a logit (or logistic regression) model to estimate the probability of participation in a recreational activity, and a negative binomial model to estimate the frequency of participation. The logit model has the general form:

$$
y=\ln (O)=\ln \left(\frac{p}{1-p}\right)=\beta_{0}+\beta^{T} X
$$

here, $p$ is the probability of participation and $y$ is the logarithm of the odds $O=\left(\frac{p}{1-p}\right) . \beta_{0} \in \mathbb{R}$ and $\beta \in \mathbb{R}^{n}$ are logit coefficients. $X \in \mathbb{R}^{n}$ is is vector of $n$ characteristics (e.g. water clarity, number of summer days, etc.) that affect the odds of participation in the recreational activity. The 


$$
\begin{aligned}
O^{A} & =\exp \left(y^{A}\right) \\
& =\exp \left(\beta_{0}^{A}+\left(\beta^{A}\right)^{T} X\right)
\end{aligned}
$$

For a change in the Secchi depth, the odds ratio $\left(\mathrm{OR}^{A}\right)$ of an activity is given by:

$$
\mathrm{OR}^{A}=\frac{O_{2}^{A}}{O_{1}^{A}}=\exp \left(\beta_{1}^{A} \Delta X_{1}\right)
$$

where $\Delta X_{1}$ is the change in Secchi depth (in meters) and $\beta_{1}^{A}$ is the corresponding logit coefficient. $O_{1}^{A}$ and $O_{2}^{A}$ are the odds of participation in an activity $(A)$ before and after the Secchi depth decreases respectively.

Table 1: Logit and negative binomial coefficients for water recreational activities with respect to water clarity (based on the hurdle model by Vesterinen et al. ${ }^{20}$ )

\begin{tabular}{l|ll|ll} 
Independent Variable & $\begin{array}{l}\text { Swimming } \\
\text { Logit }\left(\beta_{1}^{S}\right)\end{array}$ & Negbin $\left(\gamma_{1}^{S}\right)$ & $\begin{array}{l}\text { Fishing } \\
\text { Logit }\left(\beta_{1}^{F}\right)\end{array}$ & Negbin $\left(\gamma_{1}^{F}\right)$ \\
\hline Water Clarity & -0.006 & 0.059 & 0.107 & 0.097
\end{tabular}

Next, we quantify the change in frequency of participation in recreational activities using the negative binomial model and the associated coefficients (Table 1) reported in Vesterinen et al. ${ }^{20}$. The ratio of the frequency of participation in an activity $A$ is given by the "Incidence rate ratio" 
$\left(\operatorname{IRR}^{A}\right)$ :

$$
\operatorname{IRR}^{A}=\frac{\mu_{2}^{A}}{\mu_{1}^{A}}=\exp \left(\gamma_{1}^{A} \Delta X_{1}\right)
$$

here $\mu_{1}^{A}$ and $\mu_{2}^{A}$ are the rates (or frequencies) of participation and $\gamma_{1}^{A}$ is the negative binomial coefficient for an activity with respect to change in Secchi depth.

Once the impacts on the probability and frequency of participation are calculated, we estimate the annual loss in recreation trips $\left(\Omega^{A}\right)$ for an activity $A$, given population size $N$ :

$$
\Omega^{A}=N \times\left(O_{1}^{A}-O_{2}^{A}\right) \times\left(\mu_{1}^{A}-\mu_{2}^{A}\right)
$$

Using this information, we estimate the loss in recreational activities by using the cost per trip data $\left(\delta^{A}\right)$ :

$$
\text { Loss in Revenue }=\sum_{a \in \mathcal{A}} \Omega^{a} \times \delta^{a}
$$

In case of fishing and swimming, Kaval and Loomis ${ }^{24}$ estimate the value of $\delta^{A}$ to be on average 63.27 USD/trip and 57.27 USD/trip respectively (converted to 2018 USD).

\section{Cleanup Expenses}

In cases when excess nutrients are already introduced in the waterbodies, mitigation and restoration technologies are required to prevent the manifestation of nutrient problems and algae blooms. Common treatment technologies include aeration systems, alum treatment, biomanipulation, dredging, herbicide treatment, and hypolimnetic treatment. More details on these technologies and corresponding cost estimates can be found in U.S. Environmental Protection Agency ${ }^{22}$. In areas where the affected waterbody is a source for drinking water, clean up procedures such as alum treatment are required to make the water potable. The alum treatment costs are based on acres of the water surface treated. Wisconsin Department of Natural Resources ${ }^{23}$ reports the alum treatment cost to range between 344 and 861 USD/acres plus a fixed cost of 25,000 USD. We note that in some instances the clean up expenses may be higher than it would be worth to the affected community to 
live with the degraded waterbody. Our methodology to estimate the economic impacts of nutrient runoff provides a systematic way to compare these two expenses.

\section{Human and Pet Health}

Direct contact with waterbodies that are affected by HABs, either by swimming or other recreational activities, can cause illness in humans and animals. Common symptoms include dermal rashes, respiratory irritation, gastrointestinal distress, and cold/flu-like illness symptoms. Many of the health related costs of HABs are realized in the U.S. coastal states. Hoagland et al. ${ }^{17}$ estimate the cost of respiratory illnesses associated with the red tides in Saratosa County, Florida to range from 0.02 to 0.13 million USD annually. The authors use a statistical exposure-response model that is based on number of hospital emergency department visits for respiratory illness and the occurrence of algae blooms. For our case study, the HABs related health care costs in the State of Wisconsin are minor. ${ }^{23}$ Thus, the associated costs are not included in our analysis.

\section{Waste Processing}

One strategy to prevent phosphorus runoff (and HABs in turn) is by processing livestock waste and recovering phosphorus. We consider three technology variations in our case study (Figure 1). These technologies capture the different levels of complexity (ranging from simple mechanical separation to the more advanced chemical treatment) commonly employed for waste processing. The first pathway uses a screw press to separate the livestock waste into solid and liquid fractions. The solid fraction can be used as a crop fertilizer..$^{25}$ The second pathway further processes the solid fraction through granulation technology to recover $\mathrm{P}$ in the pellet form. ${ }^{26}$ The third pathway processes the liquid fraction and recovers $P$ through struvite formation. The economic viability of these waste processing pathways depends strongly on the composition of waste streams (which is highly variable), economies of scale, and transportation costs. Also, the logistical issues associated with waste collection and transportation hinder the large scale deployment of waste treatment technologies. Diverse government regulations and incentives to promote waste treatment have not been 
able to overcome these techno-economic and logistical issues. As a result, the infrastructure for waste management is at present fragmented and limited, posing a significant obstacle to mitigate the pollution of water, land, and air resources. This obstacle also hinders the sustainable growth of urban, agricultural, and food sectors.

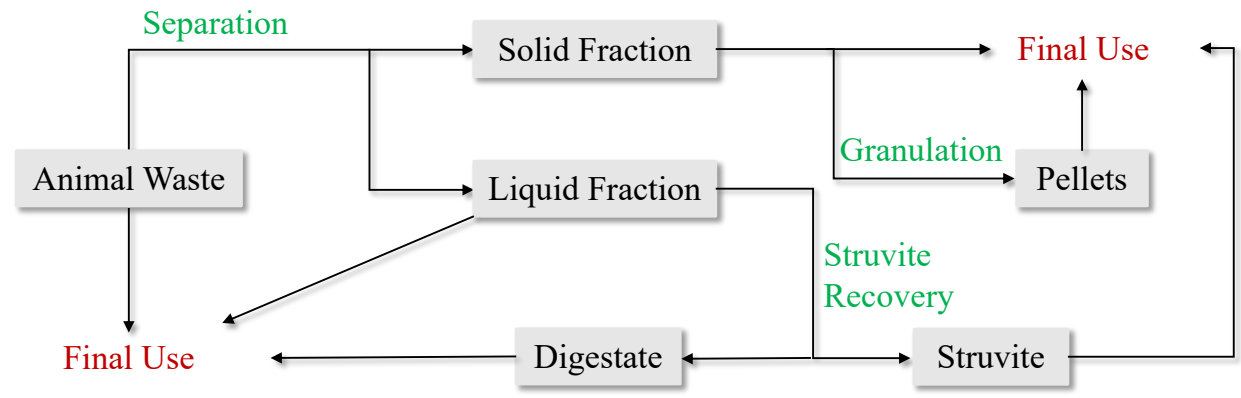

Figure 1: Different processing options for livestock waste

In this complex decision decision-making environment where a large number of stakeholders rely on shared and constrained infrastructures, a coordinated management framework ${ }^{27}$ can enable efficient exchange of products. This framework can capture complex spatio-temporal dependencies and externalities (e.g., weather). In this system, the suppliers and consumers provide bids for waste and derived products. The technology and transportation providers also submit bids for their services. An independent system operator (ISO) uses this bid information and runs a dispatch system that finds optimal physico-chemical transformation and transportation pathways that balance demands and supplies in a given geographical region (Figure 2). This approach also ensures that system-wide transportation and transformation capacities are met by the dispatch solution. The management system functions as a coordinated market that generates prices for each waste type and derived product at every location in the study area. This framework helps us determine how economic impacts of HABs can incentivize waste transportation and processing technologies. We provide a brief overview of this coordination markets model in the next section. More details about this framework and the economic properties satisfied by the cleared prices can be found in Sampat et al. ${ }^{27}$. 


\section{Coordinated Market Model}

We consider a system comprising of geographical locations/nodes $\mathcal{N}$ (e.g. dairy farms, croplands, external companies), products $\mathcal{P}$ (e.g. waste, pellets, struvite), suppliers $\mathcal{S}$ (e.g. dairy farms), consumers $\mathcal{D}$ (e.g. croplands, external companies), transportation providers $\mathcal{L}$ (e.g. hauling and shipping companies), and transformation (technology) providers $\mathcal{T}$ (e.g. mechanical separation, granulation, struvite recovery). The market players and the associated set notations are summarized in Figure 3. Each supplier $i \in \mathcal{S}$ has an associated supply flow $s_{i} \in \mathbb{R}_{+}$, location $n(i) \in \mathcal{N}$, product type $p(i) \in \mathcal{P}$, maximum offered capacity $\bar{s}_{i} \in \mathbb{R}_{+}$, and bidding cost $\alpha_{i}^{s} \in \mathbb{R}_{+}$. Each consumer $j \in \mathcal{D}$ has an associated demand flow $d_{j} \in \mathbb{R}_{+}$, location $n(j) \in \mathcal{N}$, product type $p(j) \in \mathcal{P}$, maximum requested capacity $\bar{d}_{j} \in \mathbb{R}_{+}$, and bidding cost $\alpha_{j}^{d} \in \mathbb{R}_{+}$.

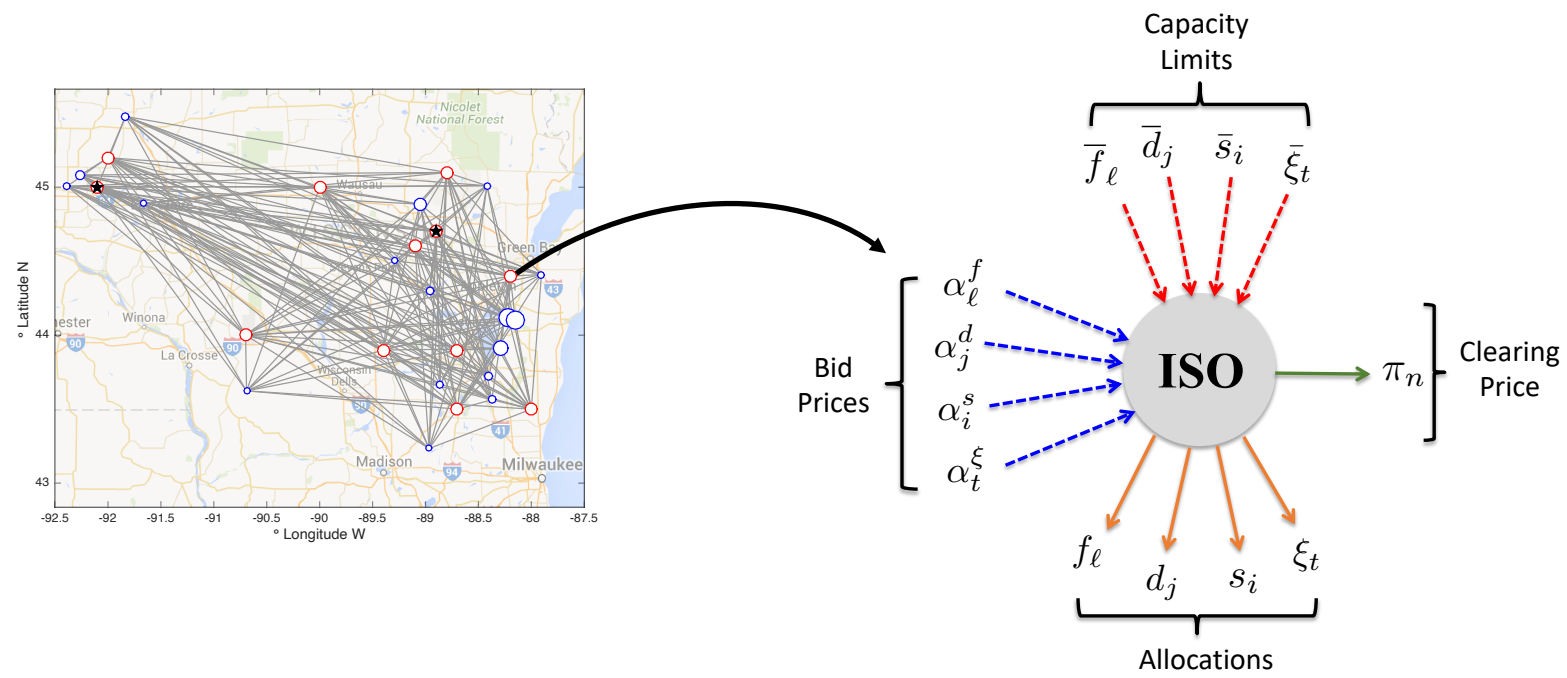

Figure 2: For every node in the supply chain network, the ISO (independent system operator) accepts bid prices and capacity limits from the market players (e.g. farmers, fertilizer consumers, federal and state agencies etc.). The ISO then solves the market clearing problem (Equations 9) to find the clearing prices of the services and the corresponding service allocations.

Each transportation provider $\ell \in \mathcal{L}$ has an associated flow $f_{\ell} \in \mathbb{R}_{+}$, sending node $n_{s}(\ell) \in \mathcal{N}$, receiving node $n_{r}(\ell) \in \mathcal{N}$, product transported $p(\ell) \in \mathcal{P}$, maximum capacity $\bar{f}_{\ell} \in \mathbb{R}_{+}$, and bidding $\operatorname{cost} \alpha_{\ell}^{f} \in \mathbb{R}_{+}$. The bidding cost is the cost of moving a unit of flow from the source to the destination node. The set of all flows entering node $n \in \mathcal{N}$ is $\mathcal{L}_{n}^{i n}:=\left\{\ell \mid n_{r}(\ell)=n\right\}$ and the set of all flows leaving node $n \in \mathcal{N}$ is $\mathcal{L}_{n}^{\text {out }}:=\left\{\ell \mid n_{s}(\ell)=n\right\}$.

Each transformation provider $t \in \mathcal{T}$ has corresponding transformation/yield factors $\gamma_{t, p} \in \mathbb{R}$, 
location $n(t) \in \mathcal{N}$, a reference product $p(t) \in \mathcal{P}$, processing capacity $\bar{\xi}_{t} \in \mathbb{R}_{+}$, and processing cost $\alpha_{t}^{\xi} \in \mathbb{R}_{+}$. Transformation factors capture the units of product $p$ consumed/generated per unit of reference product $p(t)$ consumed/generated by the technology unit. We follow the convention that $\gamma_{t, p}>0$ if product $p$ is generated, $\gamma_{t, p}<0$ if product $p$ is consumed, and $\gamma_{t, p}=0$ if product $p$ is neither produced nor consumed by the technology $t$. We note that $\gamma_{t, p(t)}=-1$ represents that one unit of reference product is consumed to produce/consume other products. For each technology, $\xi_{t} \in \mathbb{R}_{+}$represents the extent of transformation, which is the total amount of $p(t)$ processed.

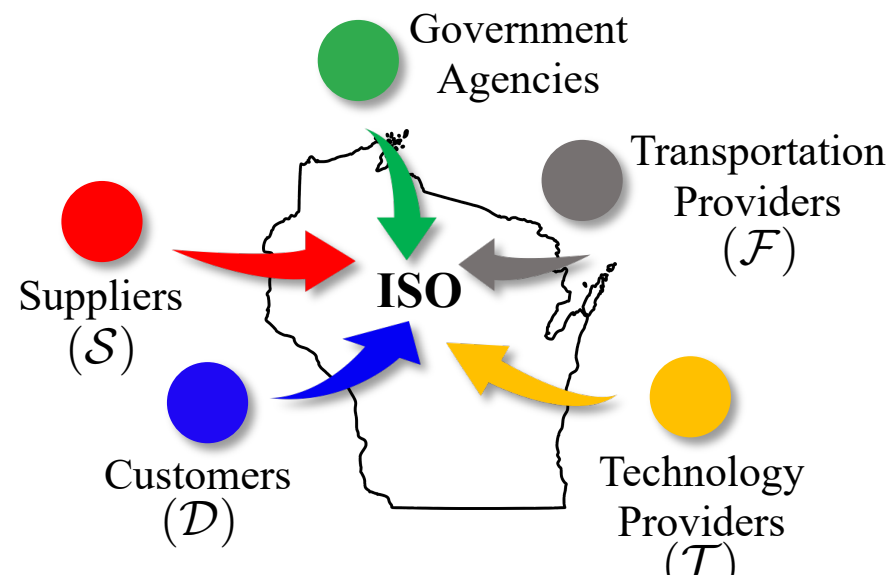

Figure 3: Market players and the corresponding mathematical set notations indicated in parenthesis. The market players submit bid prices and capacity limits for their services to the ISO.

The Environment as a Stakeholder: To capture the economic impact of HABs resulting from excess $\mathrm{P}$ in the region (denoted by $\theta_{j}$ ), we define the environment as one of the stakeholders (represented by set $\mathcal{D}^{\prime} \subset \mathcal{D}$ ). The idea being that, if there is excess $\mathrm{P}$ in the region, it can be released to the environment but at a cost $(\lambda)$. This cost can be seeing as a tipping cost or a value of service (VOS) that the environment charges society. The VOS captures the economic impacts of nutrient pollution (and HABs), which include both external costs borne by local economies and communities impacted by environmental and human effects. The VOS for this case study, based on the analysis presented in the earlier section, is set to $74.5 \mathrm{USD} / \mathrm{kg}$ excess $\mathrm{P}$. As it will become clear later, this VOS value acts as an incentive that exerts sufficient socio-economic pressure to activate a waste management market. 
The ISO uses bidding information $\left(\alpha^{d}, \alpha^{s}, \alpha^{f}, \alpha^{\xi}\right)$ and capacity limits $(\bar{d}, \bar{s}, \bar{f}, \bar{\xi})$ to solve the clearing problem (9). The model outputs are allocations $(d, s, f, \xi)$ that maximize the social welfare (9a), satisfy the physical conservation laws (9b), and capacity limits (9e)-(9h). Maximizing the social welfare function ensures that the demand served is maximized and the costs of supply, transformation and transportation are minimized. The conservation laws/balancing constraints serve as market clearing constraints that balance demand and supply at every location. The first term in parenthesis in the balancing constraint $(9 \mathrm{~b})$ is the total input flow of product $p$ into node $n$ (consisting of supply and transportation flows entering the node). The second term in parenthesis is the total output flow of product $p$ from node $n$ (consisting of the demand and transportation flows leaving the node). The third term captures the generation/consumption of product $p$ in all technologies located at node $n$

$$
\begin{aligned}
& \max _{(s, d, f, \xi)} \sum_{j \in \mathcal{D}} \alpha_{j}^{d} d_{j}-\sum_{i \in \mathcal{S}} \alpha_{i}^{s} s_{i}-\sum_{\ell \in \mathcal{L}} \alpha_{\ell}^{f} f_{\ell}-\sum_{t \in \mathcal{T}} \alpha_{t}^{\xi} \xi_{t}-\lambda \sum_{j \in \mathcal{D}^{\prime}, p(j)=P} \theta_{j} \\
& \text { s.t. }\left(\sum_{i \in \mathcal{S}_{n, p}} s_{i}+\sum_{\ell \in \mathcal{L}_{n, p}^{\text {in }}} f_{\ell}\right)-\left(\sum_{j \in \mathcal{D}_{n, p}} d_{j}+\sum_{\ell \in \mathcal{L}_{n, p}^{\text {out }}} f_{\ell}\right)+\sum_{t \in \mathcal{T}_{n}} \gamma_{t, p} \xi_{t}=0,(n, p) \in \mathcal{N} \times \mathcal{P},\left(\pi_{n, p}\right) \\
& \theta_{j} \geq d_{j}-\bar{d}_{j}, j \in \mathcal{D}^{\prime}, p(j)=P \\
& \theta_{j} \geq 0, j \in \mathcal{D}^{\prime}, p(j)=P \\
& 0 \leq d_{j} \leq \bar{d}_{j}, j \in \mathcal{D} \\
& 0 \leq s_{i} \leq \bar{s}_{i}, i \in \mathcal{S} \\
& 0 \leq f_{\ell} \leq \bar{f}_{\ell}, \ell \in \mathcal{L} \\
& 0 \leq \xi_{t} \leq \bar{\xi}_{t}, t \in \mathcal{T} .
\end{aligned}
$$

We define $\mathcal{C}$ as the set of all possible/feasible allocations $(d, s, f, \xi)$ that satisfy the capacity constraints (9e)-(9h). The dual variables $\pi_{n, p}$ of the conservation laws (9b) set values for products at different geographical locations and act as the market clearing prices. Because of this, $\pi_{n, p}$ are also referred as the locational marginal prices or nodal prices. We use the short-hand notation $\pi$ to denote all 
dual variables. Prices and allocations derived from the clearing formulation establish fundamental economic properties of the market. These prices and allocations remunerate providers (e.g., dairy farmers) and charge consumers (e.g., croplands and product buyers). Moreover, the prices are also known as coordination prices as they can be used as incentives to promote coordination between stakeholders. The allocations and prices generated from the clearing formulation also represent a competitive economic equilibrium that maximizes the collective profit of the market players ${ }^{27}$.

\section{Case Study}

We consider the upper Yahara watershed region in the State of Wisconsin (Figure 4) to reduce the occurrence of harmful algae blooms in Lake Mendota. Heavy use of livestock manure and agricultural fertilizers have resulted in excess amounts of phosphorus being accumulated in this area. The accumulated phosphorus $(\mathrm{P})$ is often washed into waterways, due to rain and snow melt. This runoff leads to the blue-green algae blooms in Lake Mendota. ${ }^{28}$ In this work, we quantify the economic impacts associated with algae blooms in Lake Mendota.

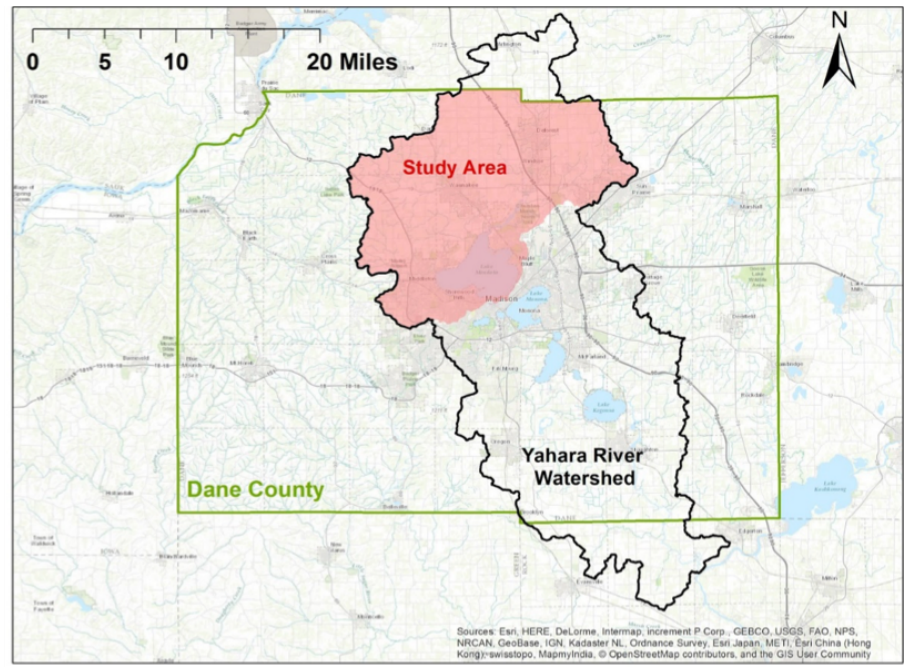

Figure 4: Lake Mendota in the Upper Yahara watershed region in Dane County, WI is the study area for quantifying the economic impacts of nutrient runoff.

The study area consists of 203 farms (148 dairy farms and 55 beef farms). These farms account for more than $99 \%$ of the P generation associated with livestock waste. Here, we consider that all 
the farms within the study area spread the waste on the associated croplands. This corresponds to spreading of 1.34 million tons of waste annually, resulting in a P release rate of 917.83 tons/yr. The croplands in our study area have a total P uptake capacity of 629.74 tons/yr. ${ }^{27}$ There is thus a surplus of 288.09 tons /yr of P. We consider that $10 \%$ of this excess P runs off to Lake Mendota. To keep the calculations on a conservative side, we have assumed that $10 \%$ of excess P runs off to the lake instead of the $10 \%$ of applied P (which is the number used in state-of-the-art LCA methods like $\mathrm{ReCiPe}^{29}$ for mid-point and end-point environmental impact categories). The initial TP concentration of Lake Mendota is considered to be $53 \mu \mathrm{g} / L$ (based on the average TP concentration for the years 2014 - 2017). ${ }^{30}$ Due to the P runoff from the overapplication of waste, we estimate (by mass balance calculations) the TP concentration of Lake Mendota increases to $110 \mu \mathrm{g} / \mathrm{L}$ (considering the lake volume to be 505 million $\mathrm{m}^{331}$ ). This increase in TP concentration acts as a basis for our calculations for quantifying the economic impacts associated with algae blooms.

\section{Economic Impacts of Algae Blooms in the Upper Yahara Watershed Region}

\section{Property Values}

When the initial TP concentration in Lake Mendota is $53 \mu \mathrm{g} / \mathrm{L}$, the Secchi depth is $0.97 \mathrm{~m}$ (by Equation 1). After P runoff, when the TP concentration of the lake increases to $110 \mu g / L$, the Secchi depth decreases to $0.64 \mathrm{~m}$. This $0.34 \mathrm{~m}$ decrease in Secchi depth corresponds to an estimated $5.3 \%$ reduction in all property values on the Lake Mendota shoreline (according to Dodds et al. ${ }^{9} 1 \mathrm{~m}$ reduction in Secchi depth reduces property values by 15.3\%). Lake Mendota has a shoreline length of 33.8 $\mathrm{km} .{ }^{32}$ Assuming an average lot length of $54.64 \mathrm{~m},{ }^{23}$ there are 619 lots on the lakeshore. We consider $85 \%{ }^{23}$ of these lots are private properties, and have a median property value of 269,100 USD. ${ }^{33}$ The reduction in Secchi depth results in a total loss of 7.46 million USD/yr. This is equivalent to 25.9 USD $/ \mathrm{kg}$ excess P released. 


\section{Recreational Cost}

In our case study for the Upper Yahara watershed region, the Secchi depth decreases by $0.34 \mathrm{~m}$. The impact of a reduction in Secchi depth on the frequency of participation in fishing and swimming is summarized in Table 2. For the current odds of participation, a survey by the Wisconsin Department of Natural Resources ${ }^{34}$ reports that $37.4 \%$ of residents participate in freshwater fishing and $41.7 \%$ swim in lakes. The current odds for fishing and swimming are thus 0.60 and 0.72 , respectively. Using the Equation 5 and the logit coefficients from Table 1, the new odds for fishing and swimming are 0.58 and 0.72 respectively. This corresponds to a new participation of $36.6 \%$ and $41.7 \%$ in fishing and swimming respectively. In case of fishing, the participation reduces by $0.8 \%$ while there is no change observed in case of swimming. There is no impact on the participation probability in swimming because the logit coefficient $\left(\beta_{1}^{S}\right)$ estimated by Vesterinen et al. ${ }^{20}$ is close to zero (Table 1).

Table 2: Impact of reduction in Secchi depth (of $0.34 \mathrm{~m}$ ) on the probability of participation in fishing and swimming in Lake Mendota.

\begin{tabular}{c|c|c} 
Activity & Fishing & Swimming \\
\hline Current Participation & $37.4 \%$ & $41.7 \%$ \\
\hline Current Odds $\left(O_{1}^{A}\right)$ & 0.60 & 0.72 \\
\hline New Odds $\left(O_{2}^{A}\right)$ & 0.58 & 0.72 \\
\hline New Participation & $36.6 \%$ & $41.7 \%$ \\
\hline Loss in Participation & $0.8 \%$ & $0 \%$
\end{tabular}

Wisconsin anglers participate in 17.3 days ${ }^{35}$ of fishing annually, while the frequency of swimming trips (by Wisconsin residents) is considered to be 5 days. ${ }^{23}$ Using these frequencies of participation and the negative binomial coefficients listed in Table 1, we estimate that a decrease in Secchi Depth of $0.34 \mathrm{~m}$ reduces the frequency of participation in fishing and swimming to 16.8 and 4.9 days respectively (by Equation 6). These results are summarized in Table 3.

Table 3: Impact of reduction in Secchi depth (of $0.34 \mathrm{~m}$ ) on the frequency of participation in fishing and swimming in the Upper Yahara watershed region.

\begin{tabular}{c|c|c} 
Activity & Fishing (days/yr) & Swimming (days/yr) \\
\hline Current Frequency $\left(\mu_{2}^{A}\right)$ & 17.3 & 5 \\
\hline New Frequency $\left(\mu_{1}^{A}\right)$ & 16.8 & 4.9
\end{tabular}


After quantifying the impacts on the probabilities and the frequencies of participation, we estimate the corresponding loss in revenue (summarized in Table 4). Kaval and Loomis ${ }^{24}$ estimate the value of a day spent fishing and swimming to be on average of 63.27 USD and 57.27 USD, respectively (converted to 2018 USD). For our study area, we consider that the participants are from the Dane County, WI, which has a population of 536,416. ${ }^{36}$ We have not considered participation from non-resident anglers or swimmers in our calculations. For our study area, we estimate a total loss of 11.8 million USD/yr and 1.19 million USD/yr in fishing and swimming respectively (by Equations 7 and 8). This translates to a loss in revenue (from recreational activities) of $45.4 \mathrm{USD} / \mathrm{kg}$ excess $P$.

Table 4: Loss in revenue from recreational activities due to a decrease in Secchi depth of $0.34 \mathrm{~m}$ in Lake Mendota

\begin{tabular}{c|c|c} 
Activity & Fishing & Swimming \\
\hline Loss in Trips (trips/yr) & $1.9 \times 10^{5}$ & $2.1 \times 10^{4}$ \\
\hline Average Trip Cost (USD/trip) & 63.3 & 57.3 \\
\hline Loss in Revenue (USD/yr) & $11.9 \times 10^{6}$ & $1.2 \times 10^{6}$
\end{tabular}

\section{Clean-up Expenses}

Lake Mendota is not a source of drinking water and thus alum treatment is not performed. However, the excessive amount of phosphorus runoff in the Yahara river waterbodies over the years has resulted in high phosphorus deposition in the bed of the streams that feed into the lake. Thus, even if all the agricultural runoff was successfully prevented from entering the Yahara river waterbodies, Lake Mendota would still be prone to algae blooms for decades to come. ${ }^{37}$ The Dane County is implementing a project called Suck the Muck ${ }^{38}$ to pump out phosphorus-laden sludge from the bottom of creeks and streams to combat the toxic algae blooms. The estimated cost of this project is 12 million USD for removing 870,000 pounds of phosphorus (or $30.2 \mathrm{USD} / \mathrm{kg}$ P removed) from the streams leading to the Yahara lakes. For our case study, where the excess $\mathrm{P}$ is 288 tons/yr and $10 \%$ of this excess $\mathrm{P}$ is assumed to runoff, the annual cost of lake cleanup translates to $3.0 \mathrm{USD} / \mathrm{kg}$ excess $P$. 


\section{Summary of Economic Impacts}

We summarize the economic estimates for the impacts due to harmful algae blooms in Lake Mendota in Table 5. From our analysis, the impact on the recreational activities is the highest (45.4 USD $/ \mathrm{kg}$ excess $\mathrm{P}$ ) followed by the impact on the property values (25.9 USD/kg excess P). Overall, every excess $\mathrm{kg}$ of $\mathrm{P}$ results in an economic loss of 74.5 USD. As we demonstrate in the next section, this economic impact can be useful in designing and activating a market that facilitates the coordinated management of organic waste.

Table 5: Summary of economic impacts of excess phosphorus (resulting in HABs) in the Upper Yahara watershed region.

\begin{tabular}{c|c} 
Impacted Category & $\begin{array}{c}\text { Economic Loss } \\
\text { (USD/kg excess P) }\end{array}$ \\
\hline Property Value & 25.9 \\
\hline Recreational Activities & 45.4 \\
\hline Lake Cleanup & 3.0 \\
\hline Human and Pet Health & - \\
\hline Total Monetized Loss & $\mathbf{7 4 . 5}$
\end{tabular}

\section{Upper Yahara Coordinated Market}

In this case study, we consider the 203 livestock farms in the Upper Yahara watershed region as the suppliers of waste. The waste is categorized into beef, dairy cow, and heifer manure and initially assumed to be offered for free. Amongst these manures, dairy cow manure has the highest P concentration. ${ }^{39,40}$ As described in the Waste Processing section, the following products can be derived from manure: granulated compressed pellets, struvite, digestate, and the manure solid fraction. In this coordinated market setting, the agricultural lands are the consumers $(\mathcal{D})$ that demand raw manure, solid fraction of manure, and digested manure. The case study includes 1,167 agricultural land nodes that can be used for waste application (to fulfill nutrient requirements). The set of consumers also includes external players (located outside the region in Madison, WI or Sauk County, WI) that accept waste surplus and buy pellets, struvite, and the solid fraction of manure. We consider the demand bidding costs to be similar to the market value of 
products: $100 \mathrm{USD} /$ tonne for pelleted waste, $800 \mathrm{USD} /$ tonne for struvite, $0.05 \mathrm{USD} /$ tonne for the solid waste fraction, $0.002 \mathrm{USD} /$ tonne for the liquid waste fraction, and $0 \mathrm{USD} /$ tonne for the digestate. ${ }^{41,42}$ Location and capacity data for demand and supply nodes are obtained from Sharara et al. ${ }^{41}$, Sampat et al. ${ }^{42}$, Sharara et al. ${ }^{43}$. The supply capacity of waste for dairy farms are based on the number of cows present at the farm and the demand capacity for croplands is based on the land area and the type of crop grown. We consider transportation bidding cost of each route as $0.3 \mathrm{USD} /$ tonne- $\mathrm{km}$ for manure and digestate (in case of pellets and struvite this value is $0.15 \mathrm{USD} /$ tonne- $\mathrm{km}$ as solids are easier to transport). For simplicity, the transportation paths between nodes are assumed to be linear and that transportation bids exist to move product between all nodes in the network/market. This gives rise to a complex logistical network. The corresponding market clearing problem is a linear program with over 30 million decision variables and 0.5 million constraints. This problem can be solved with modern solution tools such as Gurobi (version 7.5.2) ${ }^{44}$ in 15 mins. All the scripts required to reproduce the results are available at https://github.com/zavalab/JuliaBox/tree/master/EconomicImpacts.

As described earlier in the Waste Processing section, we consider three different processing options for waste treatment (Figure 1). The processing costs for these technologies are $0.23 \mathrm{USD} /$ tonne raw manure for separation, $4.00 \mathrm{USD} /$ tonne raw manure for granulation, and $38.1 \mathrm{USD} /$ tonne liquid feed for stuvite recovery. ${ }^{41,42}$ We note that struvite recovery is more expensive as it involves sophisticated technology. A tradeoff exists between the processing cost and the value of the product recovered. Struvite produced is a more concentrated in $\mathrm{P}$ and valuable than pellets; while pellets is more concentrated in $\mathrm{P}$ and valuable than the manure solid fraction. In this case study, we consider 126 hypothetical technology installations to be located at large farms (having over 500 animal units). The technologies include 61 separation units, 3 granulation units, and 62 struvite recovery units. Only CAFOs (concentrated animal feeding operations) with over 1000 animal units were considered for the installation of granulation technology. The installation locations are randomly selected and shown in Figure 5.

Under this setting of market players for livestock waste management in the Upper Yahara, we apply the coordination framework (described in the Coordinated Market Model section). Attribut- 


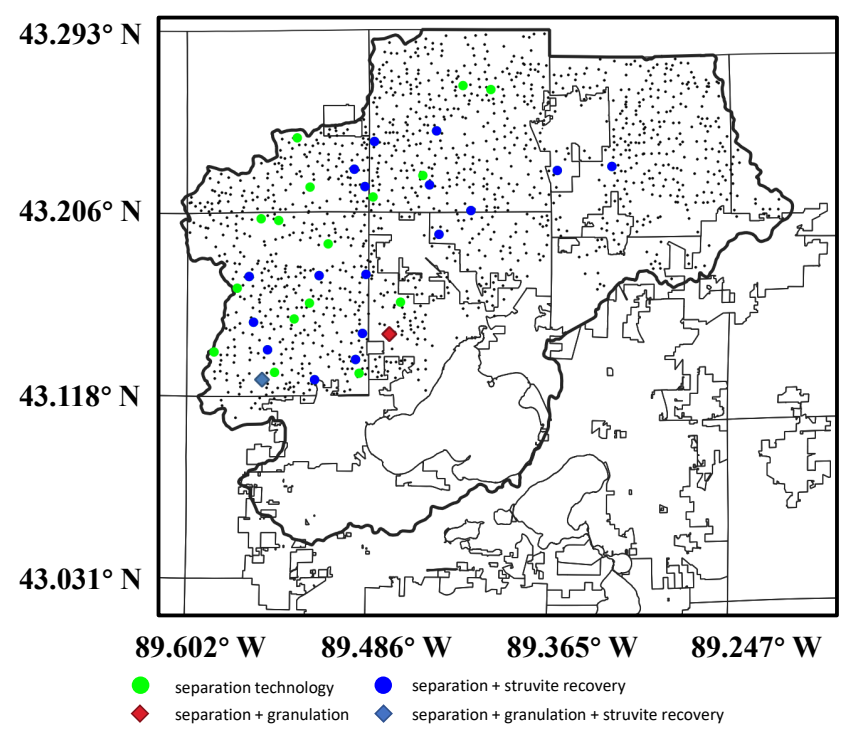

Figure 5: Locations considered in the case study for agricultural lands, farms, and waste processing technologies in the Upper Yahara watershed region. Small dots indicate location of farms and agricultural lands.

ing an economic impact (value of service VOS or $\lambda$ ) of 74.5 USD to every $\mathrm{kg}$ excess P provides an external driving force to process waste and balance the $\mathrm{P}$ in the study area (Table 6). This VOS can be provided by federal or state agencies to the dairy farmers as a part of incentives for processing waste and avoiding nutrient pollution. Under this scheme, the optimal strategy is to use separation and granulation technologies to process waste and transport the excess $\mathrm{P}$ (in the form of pellets) out of the watershed region to areas that are deficient in soil P concentration. As a result, the market model predicts that there is no excess $\mathrm{P}$ in this scenario.

Since there is uncertainty around the exact value of VOS, we perform a sensitivity analysis to study its impact on the overall $\mathrm{P}$ distribution. We observe that, in absence of an external driving force (VOS $=0 \mathrm{USD} / \mathrm{kg}$ excess $\mathrm{P})$, no waste is processed and there is $45.6 \%$ excess $\mathrm{P}$ (Figure 6 ). If the VOS value is reduced to 19 USD $/ \mathrm{kg}$ excess $\mathrm{P}$ ( $25 \%$ of the estimated value), there would still be $14.3 \%$ excess $P$ in the study area. This VOS value would only be able to activate the use of separation technologies, leaving some waste in the study area untreated. Whereas, when the VOS is 149 USD $/ \mathrm{kg}$ excess $\mathrm{P}$ (twice the estimated value), the external driving force is high enough to balance $\mathrm{P}$ in the region by using separation and granulation technologies. A VOS value of $45 \mathrm{USD} / \mathrm{kg}$ excess 
$\mathrm{P}$ is the break-even value that completely balances excess $\mathrm{P}$ in the upper Yahara watershed region. We note that, in none of these scenarios, struvite recovery technology was selected. Even though struvite has a higher market value, the high processing cost associated to this product prevents it from being economically competitive to separation and granulation technologies.

Table 6: Sensitivity analysis for different values of economic impact (or VOS).

\begin{tabular}{c|c|c}
$\begin{array}{c}\text { Economic Impact } \\
(\text { USD } / \mathrm{kg} \text { P) }\end{array}$ & $\begin{array}{c}\text { Excess P } \\
(\%)\end{array}$ & Technology Selected \\
\hline 0 & $45.6 \%$ & - \\
\hline 19 & $14.3 \%$ & Separation \\
\hline $\begin{array}{c}45 \\
\text { (break-even value) }\end{array}$ & $0 \%$ & Separation + Granulation \\
\hline $\begin{array}{c}74.5 \\
\text { (estimated value) }\end{array}$ & $0 \%$ & Separation + Granulation \\
\hline 149 & $0 \%$ & Separation + Granulation
\end{tabular}

For an economic impact of $74.5 \mathrm{USD} / \mathrm{kg}$ excess $\mathrm{P}$, the clearing prices for beef and dairy cow manure are summarized in Figure 7. Here, the clearing prices are negative, indicating that the farmers need to pay a monetary amount in order to get rid of their waste. In case of beef and dairy cow manure, the farmers need to pay on average 16.6 USD/tonne and 23.5 USD/tonne respectively. The clearing price of dairy cow manure is higher since it has more $\mathrm{P}$ concentration ${ }^{39,40}$ compared to the beef cow manure. Moreover, the clearing prices capture the geographical distribution of $\mathrm{P}$ in the study area. For the areas with higher concentration of $\mathrm{P}$, the clearing prices are more negative. These values also act as a price signal that can drive more investment in the areas with more negative clearing prices. One strategy to fund these payments can be through federal and state incentives that promote waste management practices in areas where phosphorus loading is high. This allocation of environmental cost amongst stakeholders will be analyzed in detail in our future work. 


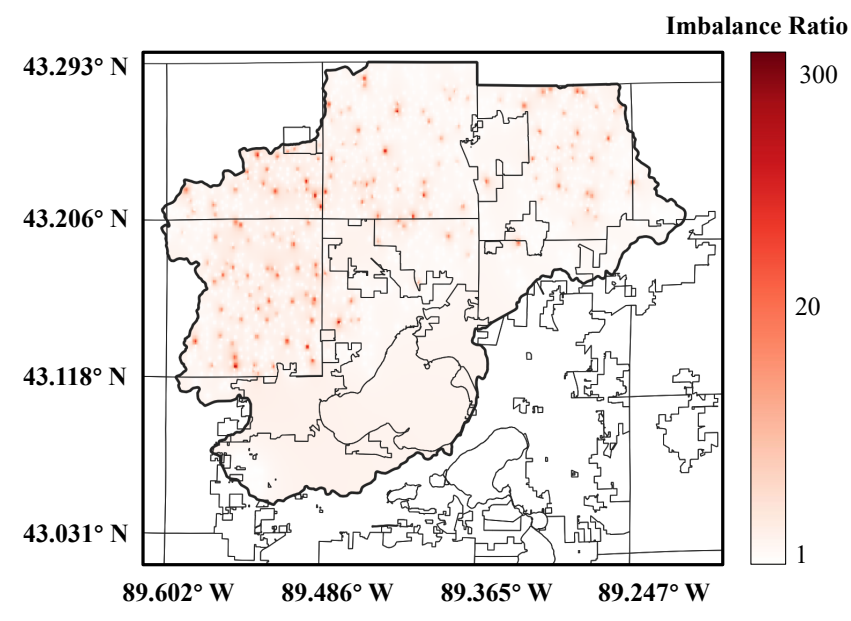

(a)

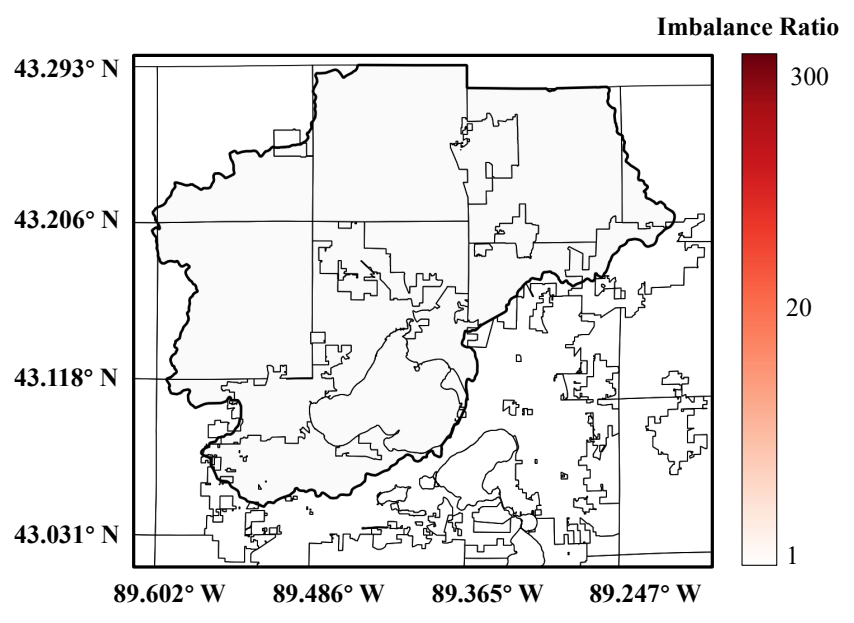

(b)

Figure 6: Phosphorus (P) imbalance maps for a value of service (VOS) of (a) 0 USD/kg excess $\mathrm{P}$ and (b) $74.5 \mathrm{USD} / \mathrm{kg}$ excess $\mathrm{P}$ in the Upper Yahara watershed region. Note that the imbalance ratio is presented in a logarithmic scale.

USD/tonne

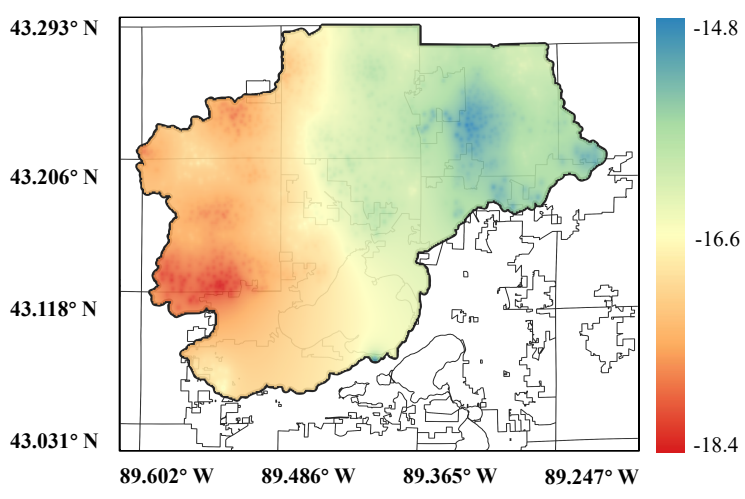

(a)

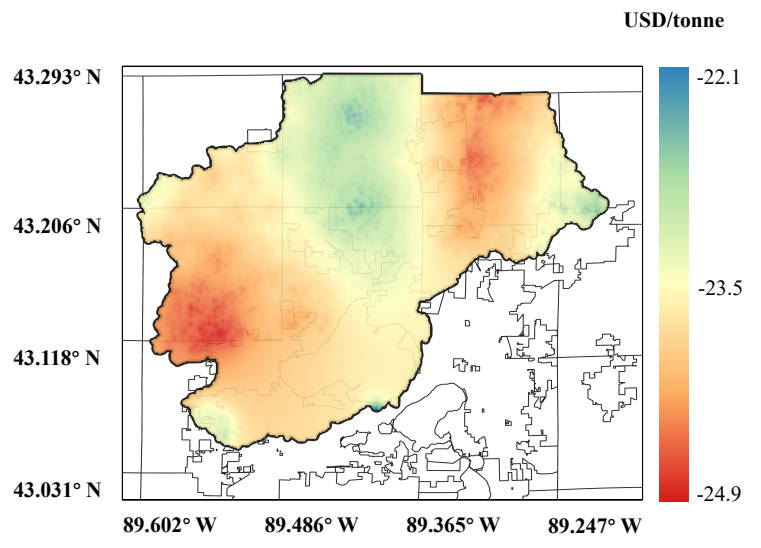

(b)

Figure 7: Clearing price for (a) beef cow manure and (b) dairy cow manure in the Upper Yahara watershed region (corresponding to a VOS of $74.5 \mathrm{USD} / \mathrm{kg}$ excess $\mathrm{P}$ ). 


\section{Conclusion}

We have presented a computational framework to estimate the economic impacts of nutrient pollution from livestock waste. It is difficult to distinguish the economic impact of nutrient pollution from that of HABs. Nonetheless an order of magnitude estimate of this impact can guide federal and state agencies to design policies and tools that reduce nutrient pollution ${ }^{22}$ which in turn causes HABs. Moreover, our methodology can capture the geographical features of nutrient pollution through the environmental cost (or VOS) and clearing prices (Figure 7). Our analysis reveals that every excess kilogram of phosphorus in the Upper Yahara watershed region results in an economic loss of 74.5 USD. In addition, we observe that for this case study the environmental cost is higher than the break-even cost to drive processing of livestock waste. Thus justifying the investment in waste processing technologies. This analysis is based on a steady state analysis and does not account the temporal system variations. Our future work will analyze the allocation of the environmental cost i.e. which stakeholder should pay for the economic loss in order to drive waste processing. One strategy to fund these payments can be through federal and state incentive programs that promote waste management practices in areas with high phosphorus imbalances.

\section{Disclaimer}

The views expressed in this article are those of the authors and do not necessarily reflect the views or policies of the U.S. Environmental Protection Agency. Mention of trade names, products, or services does not convey, and should not be interpreted as conveying, official U.S. EPA approval, endorsement, or recommendation.

\section{Acknowledgments}

We acknowledge support from the U.S. Department of Agriculture (grant 2017-67003-26055) and from the U.S. EPA (contract number EP-18-C-000016). 


\section{References}

(1) Polluted Runoff: Nonpoint Source (NPS) Pollution. U.S. Environmental Protection Agency available at https: //www.epa.gov/nps/nonpoint-source-agriculture 2019, [Online; accessed 13-August-2019].

(2) Woods Hole Oceanographic Institution, Harmful algae: Fish Kills. https://www . whoi.edu/website/redtide/impacts/wildlife/fish-kills/, [Online; accessed 01-January-2020].

(3) All 21 of Mississippi's beaches are closed because of toxic algae. CNN news available at https: //www.cnn.com/2019/07/07/us/mississippi-beaches-algae-closure-trnd/ index. htmI 2019, [Online; accessed 15-August-2019].

(4) Red tide halts shellfish harvesting all along eastern Massachusetts. CNN news available at http://thelocalne.ws/2019/07/02/ red-tide-halts-shellfish-harvesting-all-along-eastern-mass/ 2019, [Online; accessed 06-January-2020].

(5) Sea-Bird Scientific, Harmful Algae Blooms (HABs). https://www.seabird.com/ harmful-algal-bloom, [Online; accessed 06-January-2020].

(6) Bauman, A. G.; Burt, J. A.; Feary, D. A.; Marquis, E.; Usseglio, P. Tropical harmful algal blooms: An emerging threat to coral reef communities? Marine Pollution Bulletin 2010, 60, 2117-2122.

(7) Stumpf, R. P.; Tomlinson, M. C. In Remote sensing of coastal aquatic environments; Miller, R. L., Del Castillo, C. E., McKee, B. A., Eds.; Taylor \& Francis, 2005; Chapter 12, pp 277-296.

(8) What are HABs. National Oceanic and Atmospheric Administration (NOOA) available at https: //habsos.noaa.gov/about/2019, [Online; accessed 18-August-2019].

(9) Dodds, W. K.; Bouska, W. W.; Eitzmann, J. L.; Pilger, T. J.; Pitts, K. L.; Riley, A. J.; Schloesser, J. T.; 
Thornbrugh, D. J. Eutrophication of U.S. Freshwaters: Analysis of Potential Economic Damages. Environmental Science \& Technology 2009, 43, 12-19.

(10) Larkin, S. L.; Adams, C. M. Harmful algal blooms and coastal business: economic consequences in Florida. Society and Natural Resources 2007, 20, 849-859.

(11) Hoagland, P.; Scatasta, S. Ecology of harmful algae; Springer, 2006; pp 391-402.

(12) Sanseverino, I.; Conduto, D.; Pozzoli, L.; Dobricic, S.; Lettieri, T. Algal bloom and its economic impact. European Commission, Joint Research Centre Institute for Environment and Sustainability 2016,

(13) Pretty, J. N.; Mason, C. F.; Nedwell, D. B.; Hine, R. E.; Leaf, S.; Dils, R. Environmental costs of freshwater eutrophication in England and Wales. 2003.

(14) Jin, D.; Thunberg, E.; Hoagland, P. Economic impact of the 2005 red tide event on commercial shellfish fisheries in New England. Ocean E Coastal Management 2008, 51, 420-429.

(15) Hoagland, P.; Anderson, D. M.; Kaoru, Y.; White, A. The economic effects of harmful algal blooms in the United States: estimates, assessment issues, and information needs. Estuaries $2002,25,819-837$.

(16) Fleming, L. E. et al. Initial evaluation of the effects of aerosolized Florida red tide toxins (brevetoxins) in persons with asthma. Environmental Health Perspectives 2005, 113, 650-657.

(17) Hoagland, P.; Jin, D.; Polansky, L. Y.; Kirkpatrick, B.; Kirkpatrick, G.; Fleming, L. E.; Reich, A.; Watkins, S. M.; Ullmann, S. G.; Backer, L. C. The costs of respiratory illnesses arising from Florida Gulf Coast Karenia brevis blooms. Environmental Health Perspectives 2009, 117, 12391243.

(18) Phaneuf, D. J.; von Haefen, R. H.; Mansfield, C.; Van Houtven, G. Measuring Nutrient Reduction Benefits for Policy Analysis Using Linked Non-market Valuation and Environmental Assessment Models: Final Report on Stated Preference Surveys. U.S. Environmental Protection Agency 2013, 
(19) Sena, M.; Morris, M. R.; Seib, M.; Hicks, A. An exploration of economic valuation of phosphorus in the environment and its implications in decision making for resource recovery. Water Research 2020, 172, 115449.

(20) Vesterinen, J.; Pouta, E.; Huhtala, A.; Neuvonen, M. Impacts of changes in water quality on recreation behavior and benefits in Finland. Journal of Environmental Management 2010, 91,984994.

(21) Lillie, R. A.; Graham, S.; Rasmussen, P. W. Trophic state index equations and regional predictive equations for Wisconsin lakes; Bureau of Research, Wisconsin Department of Natural Resources, 1993.

(22) U.S. Environmental Protection Agency, A compilation of cost data associated with the impacts and control of nutrient pollution; 2015.

(23) Wisconsin Department of Natural Resources, Phosphorus Reduction in Wisconsin Water Bodies: An Economic Impact Analysis; 2012.

(24) Kaval, P.; Loomis, J. Updated outdoor recreation use values with emphasis on National Park recreation. Final Report, Cooperative Agreement 2003, 1200-99.

(25) Aguirre-Villegas, H. A.; Larson, R. A.; Sharara, M. A. Anaerobic digestion, solid-liquid separation, and drying of dairy manure: Measuring constituents and modeling emission. Science of The Total Environment 2019, 696, 134059.

(26) Sharara, M. A.; Runge, T.; Larson, R.; Primm, J. G. Techno-economic optimization of community-based manure processing. Agricultural Systems 2018, 161, 117-123.

(27) Sampat, A. M.; Hu, Y.; Sharara, M.; Aguirre-Villegas, H.; Ruiz-Mercado, G.; Larson, R.; Zavala, V. M. Coordinated Management of Organic Waste and Derived Products. Computers $\mathcal{E}$ Chemical Engineering 2019, 128, 352-363. 
(28) Water sustainability and climate in the Yahara Watershed. University of Wisconsin-Center for Limnology available at https: / /wsC. I imnology.wisc.edu/about/watershed 2018, [Online; accessed 23-July-2018].

(29) Huijbregts, M.; Steinmann, Z.; Elshout, P.; Stam, G.; Verones, F.; Vieira, M.; Hollander, A.; Zijp, M.; Van Zelm, R. ReCiPe 2016: A harmonized life cycle impact assessment method at midpoint and endpoint level Report I: Characterization. 2016,

(30) Wisconsin Department of Natural Resources, Lake Water Quality Report 1950 - 2017. https : //dnr.wi.gov/lakes/waterquality/Station.aspx?id=133318, [Online; accessed 21-December-2018].

(31) Lathrop, R.; Carpenter, S. Phosphorus loading and lake response analyses for the Yahara Lakes, unpublished report prepared for the Yahara CLEAN project. University of WisconsinMadison. 2011.

(32) North Temperate Lakes, Lake Mendota conditions. https://lter.limnology.wisc. edu/researchsite/lake-mendota, [Online; accessed 21-December-2018].

(33) Dane County Home Prices \& Values. https://www.zillow.com/dane-county-wi/ home-values /, [Online; accessed 23-December-2018].

(34) Wisconsin Department of Natural Resources, Wisconsin Outdoor Recreation Demand; 2011.

(35) U.S. Fish and Wildlife Service, 2006 National Survey of Fishing, Hunting, and Wildlife-Associated Recreation; 2008.

(36) U.S. Census Bureau, Quick Facts: Dane County, Wisconsin. https: / /www. census.gov/ quickfacts / danecountywisconsin, [Online; accessed 24-December-2018].

(37) Land, D. C.; Department, W. R. Legacy Sediment Removal Project. https://1wrd. countyofdane.com/Legacy-Sediment-Project, [Online; accessed 20-April-2019]. 
(38) Journal, W. S. Dane County begins 'Suck the Muck' project to remove phosphorus-laden sludge from waterways. https: //madison.com/wsj/news/local/govt-and-politics/ dane-county-begins-suck-the-muck-project-to-remove-phosphorus/ article_elea2d61-cf27-580b-afe0-5b04cc9c01d5.html, [Online; accessed 14April-2019].

(39) Nennich, T.; Harrison, J.; VanWieringen, L.; Meyer, D.; Heinrichs, A.; Weiss, W.; St-Pierre, N.; Kincaid, R.; Davidson, D.; Block, E. Prediction of manure and nutrient excretion from dairy cattle. Journal of Dairy Science 2005, 88, 3721-3733.

(40) Agricultural Waste Management Field Handbook. USDA National Resources Conservation Services (NRCS) 1992,

(41) Sharara, M. A.; Runge, T.; Larson, R.; Primm, J. G. Techno-economic optimization of community-based manure processing. Agricultural Systems 2018, 161, 117-123.

(42) Sampat, A. M.; Martín-Hernández, E.; Martín, M.; Zavala, V. M. Technologies and logistics for phosphorus recovery from livestock waste. Clean Technologies and Environmental Policy 2018, $1-17$.

(43) Sharara, M.; Sampat, A. M.; Good, L. W.; Smith, A. S.; Porter, P.; Zavala, V. M.; Larson, R.; Runge, T. Spatially explicit methodology for coordinated manure management in shared watersheds. Journal of environmental management 2017, 192, 48-56.

(44) Gurobi Optimizer Reference Manual. Gurobi Optimization, LLC 2018, 